\title{
Post Tubercular Lung: Does it need A Relook?
}

\author{
Authors \\ Dr Gajendra Vikram Singh ${ }^{1}$, Dr Santosh Kumar ${ }^{2}$, Dr Komal Lohchab ${ }^{3 *}$, \\ Dr Parul Mittal ${ }^{4}$, Dr Bhanu Pratap Pandey ${ }^{4}$, Dr Amirul haqu ${ }^{4}$ \\ ${ }^{1}$ Associate Professor, Department of T.B. \& Chest Diseases, S.N. Medical College, Agra \\ ${ }^{2}$ Associate Professor, Department of T.B. \& Chest Diseases, S.N. Medical College, Agra \\ ${ }^{3}$ Senior resident, Department of T.B. \& Chest Diseases, S.N. Medical College, Agra \\ ${ }^{4}$ Junior resident, Department of T.B. \& Chest Diseases, S.N. Medical College, Agra \\ *Corresponding Author \\ Dr Komal Lohchab \\ Department of Pulmonary Medicine, S.N. Medical College, Agra UP, India
}

\begin{abstract}
Background: Long after completion of tuberculosis (TB) therapy, many patients with previous pulmonary tuberculosis (PTB) continue to experience respiratory symptoms, frequently resulting in Ventilatory defect, deterioration in quality of life and exercise capacity.

Objective: To describe the health of patients with a history of adequately treated microbiologically positive Pulmonary Tuberculosis Patients.

Methods: A cross-sectional study that was conducted in 349 successfully treated Pulmonary tuberculosis patients of age >18 years who were put on ATT between 1stQ of 2016 TO 4th Q of 2016. Patients with current culture and AFB-positive TB were excluded. Data was collected by demographic details, respiratory symptoms, comorbidities and chest radiography findings after TB treatment. Lung function impairment was defined by the presence of at least one of these three abnormalities. Radiographic abnormalities, effect on quality of life and exercise capacity were assessed by chest X-ray, St George's questionnaire and 6 minute walk test.

Results: Of a total of 160 participants included in the study, 55.68\% were male. The mean age of participants was 35.36 years. Radiological abnormality was detected in only $84 \%$ patients. Out of finally selected 160 successfully treated pulmonary tuberculosis patients pulmonary impairment was observed in $88(55 \%)$. Mixed pattern was the most common ventilatory defect $(45.45 \%)$ followed by pure obstruction (30.68\%).Mean 6 minute walk distance was 334.39(137.07) meter and total SGRO score was 53.34(21.56).

Conclusion: Post-tuberculous pulmonary function impairment is frequent phenomenon. After tuberculosis treatment, nearly half of all tuberculosis patients evolve to impaired pulmonary impairment, mainly mixed disorder. Sensitization of patient with symptoms of pulmonary impairment after tuberculosis for an earlier visit to healthcare facilities could reduce the impact of PTB on exercise capacity and health related quality of patients.
\end{abstract}

Keywords: Pulmonary Impairment, Tuberculosis, PIAT, Exercise Intolerance. 


\section{Introduction}

Tuberculosis (TB) has afflicted mankind for millennia and continues to be a major public health burden. In 2018, an estimated 10 million people contracted TB and up 1.5 million people succumbed to the disease ${ }^{[1]}$ The lungs are the most common site of TB infection, and up to $81 \%$ of reported TB cases are pulmonary TB (PTB). ${ }^{[2]}$ TB cure is defined as the sterility of body sites or specimens previously infected by Mycobacterium tuberculosis or completion of a predetermined dose and duration of TB therapy. ${ }^{[3,4]}$ A past history of pulmonary tuberculosis (TB) is a risk factor for long-term respiratory impairment. Post-TB lung dysfunction often goes unrecognized, despite its relatively high prevalence and its association with reduced quality of life. However, a substantial proportion of patients $(40-97 \%)^{[5-10]}$ continue to experience respiratory symptoms along after completion of therapy. In addition, $40-100 \%$ of patients with previous PTB (fibrosis, emphysema, bronchiectasis) are reported to have persistent chest radiographic abnormalities ${ }^{[5,10,11]}$. Older age, female sex, longer duration of symptoms prior to the commencement of therapy, poor compliance with therapy and a history of previous PTB are factors associated with respiratory symptoms and poor radiographic resolution after completion of therapy. ${ }^{[12]}$

\section{Objectives}

The objective of this study was to describe the clinical health of patients in terms of dyspnoea grading, ventilatory abnormalities, radiographic abnormalities and outcomes of patients diagnosed as Pulmonary Impairment after tuberculosis and its effect on their exercise capacity and quality of life.

\section{Methods}

This was a cross sectional observational study in patients of pulmonary tuberculosis treated under pragmatic condition in catchment area of S.N. Medical College, Agra from $1^{\text {st }}$ Q2016 to $4^{\text {th }}$ Q 2016.

\section{Participants}

All Pulmonary TB patients aged 18 years and above who were adherent to TB treatment and successfully treated for a new or recurrence within the 3 years prior to the study were consecutively included in the study. We used the register of the TB clinic to identify patients successfully treated for PTB. Remaining patients who did not turned up to our hospital were followed up at home and all contacted patients were included in the study group. Patients presenting with the chronic obstructive airway disease, asthma, interstitial lung disease, lung cancer and lung resection \& congestive heart failure prior to the TB diagnosis were excluded from the study. Patients with contraindication for the spirometric test according to the ATS/ERS recommendations were also excluded ${ }^{[13]}$. The study protocol was submitted to the institutional review board and an ethical clearance was obtained before the recruitment of participants. All patients with a current diagnosis of sputum AFB or culture positive TB were also excluded. Informed consent was taken to the all the participants.

Data Collection: Using patients' medical records and TB register; we collected demographic data, smoking history, underlying comorbidities, clinical characteristics and radiographic findings. Demographic data included, age, gender, residence, level of education. Clinical data included symptoms (cough, dyspnea, expectoration, haemoptysis, chest pain) at the time of entrance of study. Chest radiographs were examined by a radiologist and a pulmonologist both. The type of lung lesions i.e. cavities, bronchiectasis, calcification, infiltrates, fibrotic lesions and sigh of hyperinflation were identified. Fibrotic pattern was defined as lung lesions characterized by atelectasis of the upper lobe, retraction of the hilum, compensatory lower lobe hyperinflation, and mediastinal shift toward the fibrotic area ${ }^{[14]}$. The extent of lung involvement was determined using a scoring system based on the division of each lung in areas ${ }^{[15,16]}$. 
Lung function tests (PFT) were performed according to the American Thoracic Society guidelines for subject maneuver, techniques, and quality control ${ }^{[13]}$. PFTs were performed by a trained investigator with the patient in a sitting position. A maximum of 7 to 8 tests were performed by each participant. Three tests with values within $5 \%$ were defined as being acceptable, and the best of three values was used to grade pulmonary function. Forced Expiratory Volume in $1 \mathrm{~s}$ (FEV1), Forced Vital Capacity (FVC) and FEV1/FVC ratio were measured. Airflow obstruction was defined as a post bronchodilator FEV1/FVC $<70 \%$ with FVC $>80 \%$, restrictive defects as an FEV1/FVC ratio of _ $70 \%$ with an $\mathrm{FVC}<80 \%$ predicted, and mixed defects as FVC of $<80 \%$ predicted and an FEV1/FVC ratio of $<70 \%{ }^{[13,17] .}$ Lung function impairment was defined by the presence of at least one of these three abnormalities. The severity of lung function impairment was classified as per the FEV1-based criteria agreed by the European Respiratory Society (ERS) and theATS ${ }^{[13,18] \text {. }}$

Modified Borg's dyspnoea scale was applied to assess symptom of dyspnoea. Hindi version St. George's Respiratory Questionnaire (SGRQ) was also applied to assess the effect on quality of life.6MWT was done to measure exercise capacity.

Statistical Analysis: variables are reported as means (standard deviations (SDs)) and as medians (interquartile ranges (IQRs)) when skewed. Discrete data are presented as numbers and percentages.

\section{Results}

A total of 597 subjects were evaluated to participate in the study. Out of which 406 patients were of pulmonary tuberculosis and among these patients only 349 were above the age of 18 years. 129 of them were excluded (9 contraindications, 22 died, 43 were lost in follow up, 18 patients had changed regimen, 19 were not evaluated, 7 chronic respiratory diseases, 4 congestive heart failures, 7 nonacceptable spirometric curves). 220 participants were assessed for the outcome, 158 completed the treatment, 62 were cured, 32 could not be traced, 5 expired and 18 had recurrent tuberculosis. Of the 220 , finally 165 patients were evaluated either by active tracing (144) or self reported (21).5 out of these patients were active case of tuberculosis and were not aware of it. Among these 160 patients, 83 were asymptomatic and 77 were symptomatic.

When spirometric results were presented 88 patients were found to have ventilator abnormalities. Of the 88 participants finally included in the study, $55.68 \%$ were male (figure1). The median (IQR) duration of symptoms before the diagnosis of PIAT was 17 weeks. The other characteristics of the study population are shown in Table1.

\section{Lung function impairment}

Lung function impairment in this study was observed in $88(55 \%)$ successfully treated pulmonary tuberculosis patients. The mixed defect was the most common ventilator disorder (45.45\%; 40 participants) while obstructive and restrictive defects were present in $27(30.68 \%)$ and 21 (23.86\%) subjects respectively. (Graph: 1) The univariate analysis of factors associated with lung function impairment showed that duration of symptoms, phlegm, dyspnea, fibrotic pattern and radiological abnormalities were associated with lung function impairment(Table:3)

A total of $84 \%$ patients revealed radiological abnormalities, out of which $78 \%$ patients showed cavitation and $70 \%$ showed fibrotic pattern. (Graph 4).

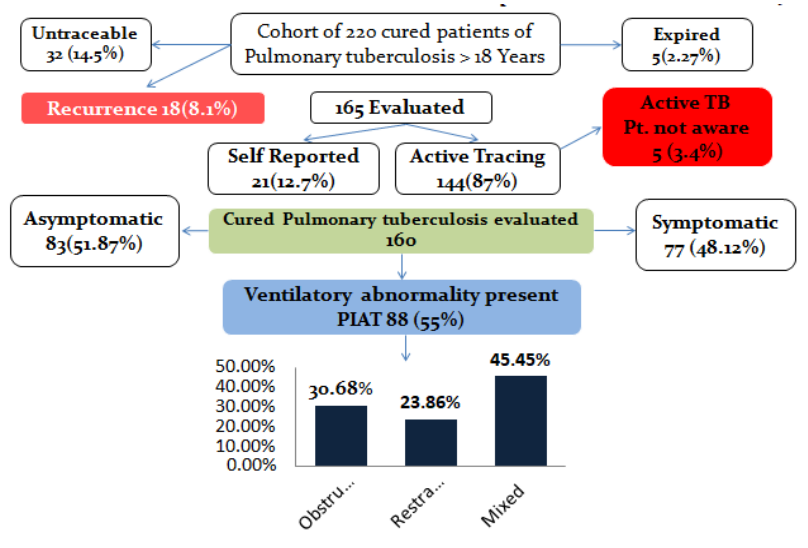


Table: 1 Demographic Profile of patients diagnosed as PIAT

\begin{tabular}{|l|c|}
\hline VARIABLES & NUMBER OF PATIENTS \% \\
\hline Gender & 55.68 \\
\hline Male & 44.32 \\
\hline Female & \\
\hline Marital status & 68.18 \\
\hline Married & 31.82 \\
\hline Unmarried & 10.22 \\
\hline Age (years) & 37.5 \\
\hline Below 20 & 11.36 \\
\hline $21-30$ & 25 \\
\hline $31-40$ & 15.9 \\
\hline $41-50$ & \\
\hline Above 50 & 28.40 \\
\hline Socio-economic status & 6.81 \\
\hline Lower & 57.95 \\
\hline Upper lower & 6.8 \\
\hline Lower middle & 0 \\
\hline Upper middle & \\
\hline Upper & 67.04 \\
\hline Living in house & 32.95 \\
\hline Crowded & \\
\hline Not crowded & 17.04 \\
\hline Smoking & 82.95 \\
\hline Smoker & 10.22 \\
\hline Non-smoker & 89.78 \\
\hline Alcohol abuse & 79.55 \\
\hline Non- alcoholic & 20.45 \\
\hline Alcoholic & \\
\hline Indoor air pollution & \\
\hline Present & \\
\hline Absent & \\
\hline & \\
\hline & \\
\hline
\end{tabular}

Table: 2 Distribution according to type of Ventilatory defect

\begin{tabular}{|l|c|c|c|c|c|c|}
\hline $\begin{array}{l}\text { Type } \\
\text { Patients }\end{array}$ & Obstruction & $\boldsymbol{\%}$ & Restriction & $\boldsymbol{\%}$ & $\begin{array}{c}\text { Mixed Ventilatory } \\
\text { defect }\end{array}$ & $\%$ \\
\hline Retreatment & 17 & 34.00 & 12 & 24.00 & 21 & 42.00 \\
\hline New & 6 & 31.57 & 6 & 31.57 & 7 & 36.84 \\
\hline Drug resistance & 4 & 21.05 & 3 & 15.7 & 12 & 63.15 \\
\hline TOTAL & 27 & 30.68 & 21 & 23.86 & 40 & 45.45 \\
\hline
\end{tabular}

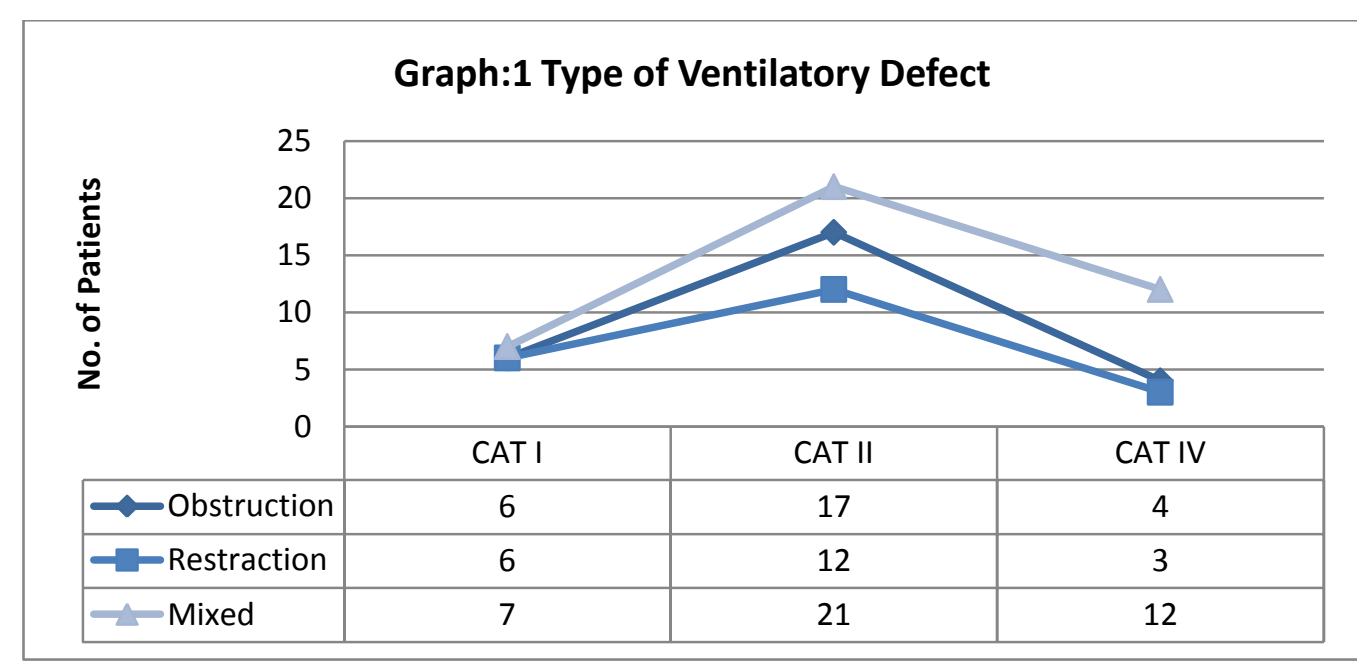


Table: 4 Distribution of patients according to presence of radiological abnormality

\begin{tabular}{|l|c|c|}
\hline $\begin{array}{l}\text { Type of anti- } \\
\text { tuberculosis treatment }\end{array}$ & $\begin{array}{c}\text { YES } \\
\%\end{array}$ & $\begin{array}{c}\text { No. } \\
\%\end{array}$ \\
\hline CAT-II & 87 & 12.4 \\
\hline CAT-I & 77.9 & 22.05 \\
\hline CAT-IV & 86.04 & 13.9 \\
\hline TOTAL & $\mathbf{8 4 . 4}$ & 15.5 \\
\hline
\end{tabular}
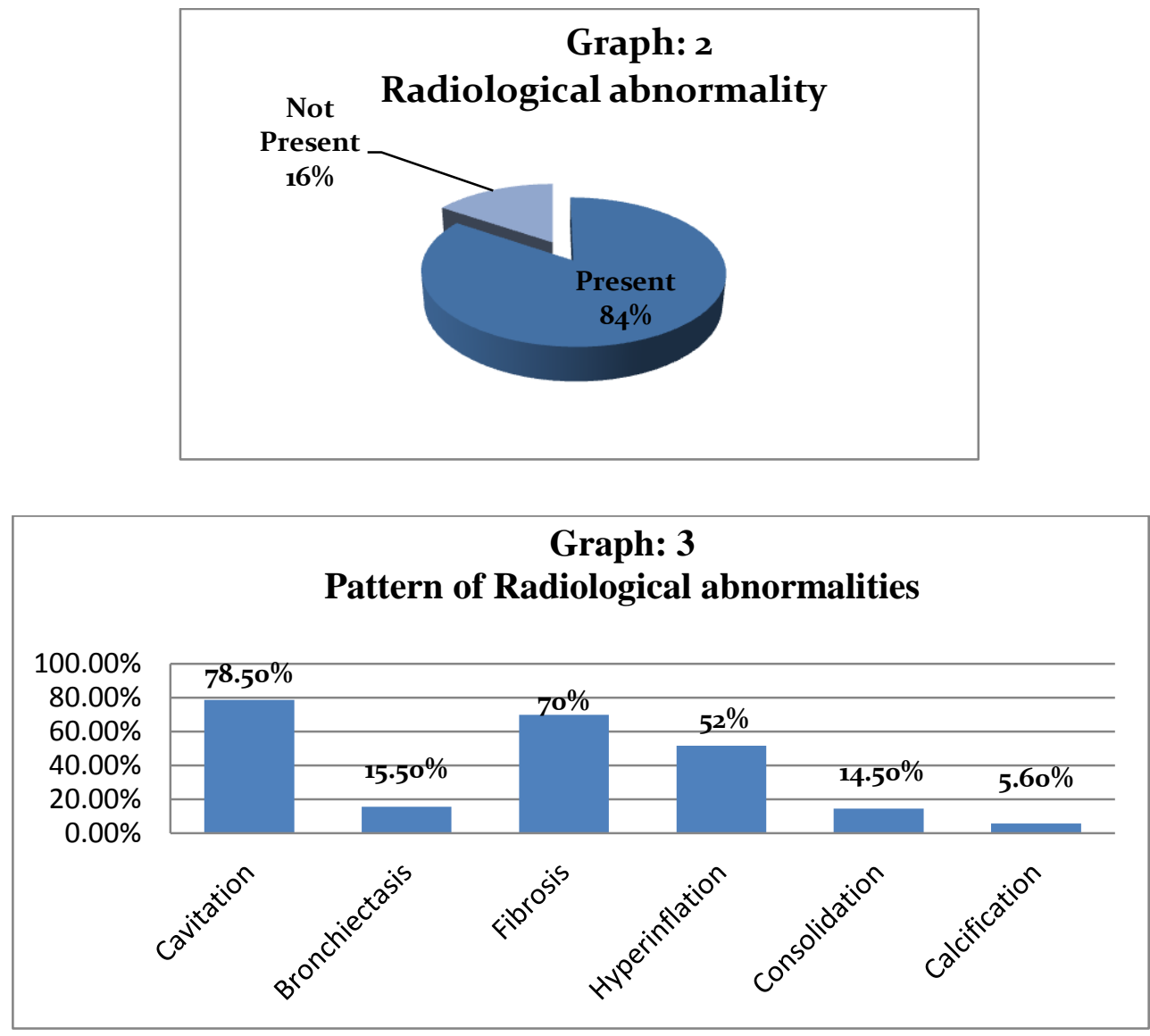

Table 5: SGQR scoring for assessment of effect on quality of life

\begin{tabular}{|l|c|}
\hline HRQL & Mean \pm SD \\
\hline SGRQ Symptom Score & $52.72(17.55)$ \\
\hline SGRQ Activity Score & $60.14(21.34$ \\
\hline SGRQ Impact Score & $49.52(26.74)$ \\
\hline Total SGRQ Score & $53.34(21.56)$ \\
\hline
\end{tabular}

Table 6: Multivariate analysis of factors associated with lung function impairment

\begin{tabular}{|l|c|}
\hline Parameters & Mean \pm SD \\
\hline BMI & $\mathbf{1 8 . 9 5 ( 3 . 1 1 )}$ \\
\hline FEV1\% & $\mathbf{5 1 . 1 2 ( 1 0 . 9 4 )}$ \\
\hline 6 Minute walk Distance & $\mathbf{3 3 4 . 3 9 ( 1 3 7 . 0 7 )}$ \\
\hline Borg Scale Score(Post 6MWT) & $\mathbf{4 . 0 5 ( ( 1 . 9 )}$ \\
\hline
\end{tabular}

\section{Discussion}

The respiratory physiological manifestations of post-TB lung disease are varied. ${ }^{[19,20]}$ In a crosssectional study by Mbatchou Ngahane et al. ${ }^{[20]}$ of 269 patients successfully treated for PTB, only
$54.6 \%$ of the cohort had normal lung function tests. The remainder of the patients had restrictive $(31.6 \%)$ or obstructive changes $(4.1 \%)$ or a mixed pattern $(5.2 \%)$. Willcox and Ferguson ${ }^{[19]}$ reported obstructive changes in $68 \%$ of their patients and 
restrictive changes in $17 \%$, while $15 \%$ had normal lung function tests. Although, the present study on post-tuberculous spirometric changes in SN Medical College showed that $45.5 \%$ of subjects with a past history of treated pulmonary TB experienced lung function impairment, with mixed pattern being the most common disorder. In a Canadian study of 25 newly diagnosed TB patients treated with DOT that correlated PFTs with computerized lung tomography, four subjects $(16 \%)$ had impaired lung function and one subject had severe impairment (an FEV1 of $<1.0$ 1) ${ }^{[21]}$. That study found that pulmonary function impairment present at the time of tuberculosis diagnosis was not significantly changed after six months of therapy ${ }^{[21]}$. A Latin American study identified impairment associated with tuberculosis from a population view found that persons reporting a prior TB diagnosis were more than twice as likely to have airflow obstruction ${ }^{[22]}$. In a retrospective study of South African men sufficiently fit to be employed as gold miners, pulmonary impairment was found to be stable 12 months after the TB diagnosis [23]. In another study from South Africa, pulmonary impairment observed in hospitalized TB patients at the beginning of treatment improved modestly but still persisted after six months of treatment ${ }^{[24]}$. A recent cross-sectional study of MDR-TB patients from India revealed that almost all (45/47 or 96\%) had abnormal PFT values, and $98 \%$ had residual radiological sequelae despite completing standardized and recommended anti-TB therapy; these findings suggest that PIAT is worse in

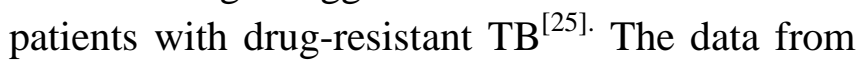
our retrospective, non-selected and racially heterogeneous cohort of tuberculosis patients treated with DOT were insufficient to establish a relationship between drug resistant $\mathrm{TB}$ and changes in PIAT. However, we were able to demonstrate that impairment identified after completion of treatment remained stable after weeks or months of observation. These findings support a recent report by the TAMI group, from Taipei, Taiwan, in which pulmonary impairment was described in a retrospective cohort of 115patients successfully treated for mycobacterial diseases including $\mathrm{TB}^{[26]}$. In that study, it was suggested that despite successful treatment residual impairments persisted. Taken together, the results from previous studies ${ }^{[21,23,22,26,25,24]}$ and the present study of patients receiving universal Anti-tubercular treatment demonstrate that, reducing PIAT requires tuberculosis prevention, not earlier tuberculosis treatment. It is intuitive that delayed tuberculosis treatment would be associated with poorer clinical outcomes ${ }^{[27,28]}$. However, data from other studies conflict with this suggestion ${ }^{[29]}$. Based on American Medical Association criteria for pulmonary impairment, $57 \%$ of patients in this cohort had PIAT. PIAT could potentially be prevented at three levels. Primary prevention could be achieved by preventing TB infection. Secondary prevention would focus on preventing persons with LTBI from developing tuberculosis. Tertiary prevention would rely on developing targeted treatments for persons with active tuberculosis to mitigate lung damage. ${ }^{[30,31,32-33]}$ Forty-five percent of our cohort had normal PFTs, and the follow-up period was relatively short. We therefore have no reason to suspect that excluding those who did not perform both tests would change our findings. Furthermore, although the study was limited to a single geographic area, the racially and ethnically heterogeneous study population suggests that our conclusions are generalizable.

In summary, we found that a substantial number of successfully treated pulmonary tuberculosis patients are prone to develop ventilatory defect and suffered from poor exercise intolerance and impaired health related quality of life. These data suggest that impairment, when present, may be permanent. It is important for both clinicians providing ongoing are for patients with a history of TB and policy makers providing tuberculosis control guidelines to recognize that the completion of tuberculosis therapy is insufficient to prevent chronic pulmonary impairment in many patients. Additionally, the impairment is 
frequently severe enough to result in disability and possibly even premature death. Taken together, the results from previous studies and the present study of patients receiving ant tuberculosis treatment demonstrate that, for many individuals, the completion of tuberculosis therapy is only the beginning of new and distressing illness.

\section{Conflict of interest}

None declared.

\section{Acknowledgements}

The authors thank the TB and Chest staff of SN Medical College for their contribution during the preparation of the manuscript. We are indebted to the participants who made this study possible.

Funding: None

\section{References}

1. World Health Organization. Global Tuberculosis Report 2019. http://www.who.int/tb/ publications/global_report/gtbr2019_execu tive_summary.pdf

2. World Health Organization. Global Tuberculosis Report 2015. 2015. http://apps.who.int/iris/ bitstream/10665/191102/1/978924156505

9_eng.pdf (accessed 25 January 2019).

3. Nahid P, Dorman SE, Alipanah N, et al. Official American Thoracic Society/Centers for Disease Control and Prevention/Infectious Diseases Society of America Clinical Practice Guidelines: Treatment of drug-susceptible tuberculosis. Clin Infect Dis 2016;63(7):e147-e195. https://doi. org/10.1093/cid/ciw376

4. Chakaya J, Kirenga B, Getahun H. Long term complications after completion of pulmonary tuberculosis treatment: A quest for a public health approach. J ClinTuberc Other Mycobact Dis 2016;3(May):10-12. https://doi.org/10.1016/j.jctube.2016.03.00 1
5. Singla N, Singla R, Fernandes S, Behera D. Post treatment sequelae of multi-drug resistant tuberculosis patients. Indian J Tuberc 2009;56(4):206-212. http://medind.nic.in/ibr/t09/i4/ibrt09i4p20 6.pdf (accessed 5 February 2019).

6. Muniyandi M, Rajeswari R, Balasubramanian R, et al. Evaluation of post-treatment health-related quality of life (HRQoL) among tuberculosis patients. Int J Tuberc Lung Dis 2007;11(8):887-892.

7. Rajeswari R, Muniyandi M, Balasubramanian R, Narayanan PR. Perceptions of tuberculosis patients about their physical, mental and social wellbeing: A field report from south India. SocSci Med 2005;60(8):1845-1853. https://doi.org/10.1016/j.socscimed.2004.0 8.024

8. Nihues S de S, Mancuzo EV, Sulmonetti $\mathrm{N}$, et al. Chronic symptoms and pulmonary dysfunction in post-tuberculosis Brazilian patients. Braz J Infect Dis 2015;19(5):492497 . https://doi.org/10.1016/j. bjid.2015.06.005

9. Ramos LMM, Sulmonett N, Ferreira CS, Henriques JF, de Miranda SS. Functional profile of patients with tuberculosis sequelae in a university hospital. J Bras Pneumol 2006;32(1):43-47. https://doi. org/10.1590/S1806-37132006000100010

10. Musafiri S, Dusabejambo V, Munganyinka BC, Manzi O, Kalisa L, Rutayisire PC. The aftermath of pulmonary tuberculosis: Predictors of severe pulmonary sequelae and quality of life of patients visiting a tertiary level of care in Rwanda, East Africa. Austin J PulmRespir Med 2015;2(2):1027. http:// austinpublishinggroup.com/pulmonaryrespiratory-medicine/fulltext/ajprm-v2id1027.php\#Title (accessed 4 February 2019).

11. Menon B, Nima G, Dogra V, Jha S. Evaluation of the radiological sequelae 
after treatment completion in new cases of pulmonary, pleural, and mediastinal tuberculosis. Lung India 2015;32(3):241$245 . \quad$ https://doi.org/10.4103/09702113.156233

12. Al-Hajjaj MS, Joharjy IA. Predictors of radiological sequelae of pulmonary tuberculosis. ActaRadiol 2000;41(6):533537.

https://doi.org/10.1080/028418500127346 153

13. M.R. Miller, J. Hankinson, V. Brusasco, F. Burgos, R. Casaburi, A. Coates, et al., Standardisation of spirometry, Eur. Respir. J. 26 (2) (2005 Aug) 319e338.

14. H.Y. Kim, K.S. Song, J.M. Goo, J.S. Lee, K.S. Lee, T.H. Lim, Thoracic Sequelae and Complications of Tuberculosis, Radiographics 21 (2001) 839e860.

15. G.L. Snider, L. Doctor, T.A. Demas, A.R. Shaw, Obstructive airway disease in patients with treated pulmonary tuberculosis, Am. Rev. Respir. Dis. 103 (5) (1971 May) 625e640.

16. P.A. Willcox, A.D. Ferguson, Chronic obstructive airways disease following treated pulmonary tuberculosis, Respir. Med. 83 (3) (1989 May) 195e198.

17. Global Initiative for Chronic Obstructive Lung Disease. Global strategy for the diagnosis, management and prevention of chronic pulmonary disease (updated 2014). Available from: http://www.goldcopd.org/uploads/users/fil es/GOLD_Report_2014_Jan23.pdf.

Accessed June 22, 2015.

18. R. Pellegrino, G. Viegi, V. Brusasco, R.O. Crapo, F. Burgos, R. Casaburi, et al., Interpretative strategies for lung function tests, Eur. Respir. J. 26 (5) (2005 Nov) 948e968

19. Willcox PA, Ferguson AD. Chronic obstructive airways disease following treated pulmonary tuberculosis. Respir Med
https://doi.org/10.1016/S0954-

6111(89)80031-9

20. Mbatchou Ngahane BH, Nouyep J, Nganda Motto M, et al. Post-tuberculous lung function impairment in a tuberculosis reference clinic in Cameroon. Respir Med 2016;114(May):67-71. https://doi. org/10.1016/j.rmed.2016.03.007

21. Long R, Maycher B, Dhar A, Mafreda J, Hershfield E, Anthonisen N. Pulmonary tuberculosis treated with directly observed therapy: serial changes in lung structure and function. Chest 1998;113(April (4)):933-43.

22. Menezes AM, Hallal PC, Perez-Padilla R, Jardin JRB, Muino A, Lopez MV, et al. Tuberculosis and airflow obstruction: evidence from the PLATINO study in Latin America. EurRespir J 2007;30(December (6)):1180-5.

23. Eva Hnizdo TSGC. Chronic pulmonary function impairment caused by initial and recurrent pulmonary tuberculosis following treatment. Thorax 2000;55:328.

24. Plit ML, Anderson R, Van Rensburg CEJ, Page-Shipp L, Blott JA, Fresen JL, et al. Influence of antimicrobial chemotherapy on spirometric parameters and proinflammatory indices in severe pulmonary tuberculosis. EurRespir J1998;12:351—6.

25. Singla N, Singla R, Fernandes S, Behera D. Post treatment sequelae of multi-drug resistant tuberculosis patients. Indian $\mathrm{J}$ Tuberc 2009;56(4):2006-12.

26. Chung KP, Chen JY, Lee CH, Huey-Dong W, Jann-Yuan W, Li-Na L, et al. Trends and predictors of changes in pulmonary function after treatment for pulmonary tuberculosis. Clinics2011;66(4):549-56.

27. Hnizdo E, Sircar K, Yan T, Harber P, Fleming J, Glindmeyer HW. Limits of longitudinal decline for the interpretation of annual changes in FEV1 in individuals. 
Occup Environ Med 2007;64(October (10)):701-7.

28. Greenaway C, Menzies D, Fanning A, Grewal R, Yuan L, FitzGerald JM, et al. Delay in diagnosis among hospitalized patients with active tuberculosis predictors and outcomes. Am J RespirCrit Care Med 2002;165:927-33.

29. Vree M, Huong NT, Duong BD, Co NV, Sy DN, Cobelens FG,et al. High mortality during tuberculosis treatment does notindicate long diagnostic delays in Vietnam: a cohort study.BMC Public Health 2007;7:210.

30. Mayosi BM, Wiysonge CS, Ntsekhe M. Clinical characteristics and initial management of patients with tuberculous pericarditis in the HIV era: the Investigation of the Management of Pericarditis in Africa (IMPI Africa) registry. BMC Infect Dis 2006;6:2.

31. Mayosi BM, Wiysonge CS, Ntsekhe M, Gumedze F, Volmink JA, Maartens G, et al. Mortality in patients treated for tuberculous pericarditis in sub-Saharan Africa. S Afr MedJ 2008;98(January (1)):36-40.

32. Thwaites GE, Nguyen DB, Nguyen HD, Hoang TQ, Do TT,Nguyen TC, et al. Dexamethasone for the treatment oftuberculous meningitis in adolescents and adults. N Engl J Med 2004;351:1741-51.

33. Pasipanodya J, Gumbo T. An oracle: antituberculosis pharmacokineticspharmacodynamics, clinical correlation, and clinical trial simulations to predict the future. Antimicrob Agent Chemother 2011;55(1):24-34. 